\title{
EFEITO DE SUBDOSES DE SULFENTRAZONE EM PLANTAS AQUÁTICAS E ECOTOXICOLOGIA PARA BIOINDICADORES
}

\section{EFFECT OF SULFENTRAZONE SUBDOSES IN AQUATIC PLANTS AND ECOTOXICOLOGY FOR BIOINDICATORS}

\author{
Tales Ribeiro da Silva ${ }^{1 *}$, Isabella Alves Brunetti² ${ }^{2}$ Luiz Henrique de Oliveira Perez ${ }^{1}$, \\ Pamela Castro Pereira ${ }^{2}$, Karina Petri dos $\operatorname{Santos}^{1}$, Claudinei da Cruz ${ }^{1}$ \\ ${ }^{1}$ Laboratório de Ecotoxicologia e Eficácia dos Agrotóxicos, LEEA, do Centro Universitário da Fundação \\ Educacional de Barretos, SP. \\ ${ }^{2}$ Universidade Estadual Paulista (UNESP), Campus Jaboticabal, Faculdade de Ciências Agrárias e \\ Veterinárias, Jaboticabal, SP. \\ *Bolsista do programa PIBIC/CNPq/Unifeb, Barretos, SP.
}

\begin{abstract}
Resumo
O objetivo deste estudo foi avaliar os efeitos de subdoses do herbicida sulfentrazone em plantas aquáticas submersas e determinar a toxidade aguda (CL/CE50) para plantas aquáticas (Azolla caroliniana, Lemna minor e Wolffia brasiliensis), caramujo (Pomacea canaliculata) e plantas terrestres (Cucumis sativus, Oryza sativa, Cucurbita spp., Zea mays, Raphanus sativus e Glycine max). Para as três plantas aquáticas, foram testadas as subdoses de $0,01,0,05,0,1,1,0,2,5$ e $5,0 \mathrm{mg} \mathrm{L}^{-1}$. Os ensaios de toxicidade aguda foram realizados com $0,1,1,07,3,5,11,16,36,5$ e 118,0 $\mathrm{mg} \mathrm{L}^{-1}$ para os organismos aquáticos A. caroliniana, W. brasiliensis, L. minor e P. canaliculata, enquanto para as plantas terrestres $O$. sativa, G. max, Cucurbita spp., Z. mays, $R$. sativus e $C$. sativus foram utilizadas as concentrações $0,01,0,06,0,1,0,7,3,5$ e 5,0 $\mathrm{mg} \mathrm{L}^{-1}$. A classificação ecotoxicológica do sulfentrazone foi de praticamente não tóxico para $A$. caroliniana, $W$. brasiliensis e $P$. canaliculata e muito tóxico para $L$. minor. Na avaliação de subdoses, ocorreu crescimento significativo para $E$. densa, diferindo das demais plantas. O pepino (C. sativus) apresentou maior sensibilidade à presença do herbicida em relação às outras plantas terrestres. Os organismos-teste L. minor (planta aquática) e C. sativus (planta terrestre) apresentaram excelente resposta de sensibilidade ao sulfentrazone. Quanto às plantas aquáticas, ocorreu estímulo de crescimento do fragmento apical para $E$. densa, o que indica possível efeito de hormese desse herbicida, sendo necessário cuidado com essa molécula para não atingir o ambiente aquático.
\end{abstract}

Palavras-chave: Bioensaio, hormese, toxicologia

\begin{abstract}
The objective of this study was to evaluate the effects of sulfentrazone herbicide underdoses for submerged aquatic plants and to determine acute toxicity (CL/CE50) for aquatic plants Azolla caroliniana, Lemna minor and Wolffia brasiliensis snail (Pomacea canaliculata), and terrestrial plants (Cucumeris sativus, Oriza sativa, Cucurbita spp., Zea mays, Raphanus sativus, and Glycine max). For the three submerged plants, the underdoses were tested of $0.01 ; 0.05 ; 0.1 ; 1.0 ; 2.5 ; 5.0 \mathrm{mg} \mathrm{L}^{-1}$. Acute toxicity tests were performed with $0.1 ; 1.07 ; 3.5 ; 11.16 ; 36.5 ; 118.0 \mathrm{mg} \mathrm{L}^{-1}$ for aquatic organisms $A$. caroliniana, $W$. brasiliensis, L. minor and P. canaliculata and for terrestrial plants, O. sativa, G. max, Cucurbita spp., Z. mays, R. sativus and C. sativus
\end{abstract}

Autor para correspondência: Tales Ribeiro da Silva, endereço: Avenida Professor Roberto Frade Monte, n. 389, Barretos, SP. E-mail: talesribeirodasilva@hotmail.com

Recebido para publicação: 10/02/2020

Aceito para publicação: 07/05/2020

https://doi.org/10.4322/1980-0029.162020 
concentrations were used with $0.01 ; 0.06 ; 0.1 ; 0.7 ; 3.5 ; 5.0 \mathrm{mg} \mathrm{L}^{-1}$. The ecotoxicological classification of sulfentrazone was practically not toxic for A. caroliniana, W. brasiliensis, and $P$. canaliculata and much toxic for $L$. minor. In the evaluation of underdoses a significant growth for $E$. densa occurred, differing from the other plants. Cucumber (C. sativus) is more sensitive than other terrestrial plants in the presence of the herbicide. The organisms test L. minor (aquatic plant) and cucumber (C. sativus) (terrestrial plant), found an excellent sensitivity response to sulfentrazone. For aquatic plants, there was apical growth stimuli for aquatic plants $E$. densa, which indicates the possible hormonal effect of this herbicide, being necessary care with this molecule to not reach the aquatic environment.

Keywords: Bioassay, hormesis, toxicology

\section{Introdução}

A intensificação da agricultura mundial tem exercido pressão sobre os seres humanos e outros organismos não alvos de aplicação, o que desafia sua capacidade de lidar e/ou prosperar sob condições estressantes (Adger et al., 2010; Fischer, 2019; Agathokleous \& Calabrese 2020).

Segundo Miranda et al. (2017), a aplicação de herbicidas para o controle de plantas daninhas tem sido uma atividade comum na agricultura global, com o objetivo de ampliar a produtividade. De acordo com Peres et al. (2017), a presença de resíduos de pesticidas, especialmente herbicida, pode ser um dos fatores que contribui para o crescimento de colonizações de plantas aquáticas. Entre os efeitos que envolvem a presença de subdoses de herbicidas nas plantas há a hormese, que é definida como uma relação dose-resposta bifásica com doses baixas, ocasionando efeitos estimulantes pela ativação de mecanismos adaptativos que aumentam a resiliência. Já em doses maiores, pode induzir inibição de respostas e, em doses elevadas, causar toxicidade (Calabrese \& Blain, 2011; Bertoncelli et al., 2018; Agathokleous et al., 2019a, 2019b).

A dinâmica ambiental desses produtos deve ser estudada, pois parte dos herbicidas não atinge o alvo prioritário de controle e afeta os organismos não alvos da aplicação. Um dos principais problemas para avaliação dos possíveis efeitos dos herbicidas no ambiente é o desenvolvimento de organismos sentinelas ou bioindicadores (Silva et al., 2012).

Entre os herbicidas que podem causar efeitos ambientais e devem ser avaliados está o sulfentrazone. Esse herbicida tem como mecanismo de ação a inibição da enzima protoporfirinogênio oxidase (PROTOX), que leva ao acúmulo da protoporfirina IX, ocasionando a peroxidação lipídica e, por consequência, a destruição das membranas celulares de plantas, além de ter mecanismo de ação sistêmica no controle de várias espécies de plantas consideradas daninhas, monocotiledôneas e dicotiledôneas (Tiburcio et al., 2012). Esse herbicida é aplicado em pré-emergência das plantas daninhas, sistêmico e seletivo.

A persistência em solos cultivados com soja, bem como os efeitos da toxicidade do resíduo em diversas culturas subsequentes, por meio de bioensaios, demonstrou que o resíduo do sulfentrazone aplicado nas doses do produto comercial de $600 \mathrm{~g}$ ia ha ${ }^{-1}$ e $1.200 \mathrm{~g}^{1}$ ia ha-1 afetou o crescimento e o rendimento das culturas de milheto (Pennisetum glaucum) e aveia (Avena sativa), mas não afetou o girassol (Helianthus annuus) e o feijão (Phaseolus vulgaris) (Blanco \& Velini, 2005). A planta aquática Pistia stratiotes mostrou baixa sensibilidade ao carfentrazone-ethyl, de mesmo mecanismo de ação do sulfentrazone, em $15 \mathrm{~g}$ ia ha $^{-1}$, porém, em 30 e $60 \mathrm{~g}^{\text {ia ha }}{ }^{-1}$, a sensibilidade foi excelente aos 7 DAA, com porcentagem de controle superior a $90 \%$ (Foloni \& Pitelli, 2005).

Para compreender os efeitos do herbicida sulfentrazone no meio ambiente, é importante determinar sua toxicidade para organismos bioindicadores aquáticos e terrestres, selecionando espécies não alvos para o monitoramento ambiental dessa molécula, além de caracterizar os possíveis efeitos de subdoses em plantas aquáticas, pois, de acordo com Agathokleous \& Calabrese (2020), estudos indicam que o efeito hormese pode melhorar a qualidade dos processos de avaliação de riscos ambientais, juntamente com outros modelos de dose-resposta. Assim, o objetivo deste estudo foi determinar os efeitos de subdoses do sulfentrazone em plantas aquáticas submersas (Egeria densa, Egeria najas e Hydrilla verticillata) e avaliar sua toxidade aguda (CE/CL50) para os bioindicadores Pomacea canaliculata, Azolla caroliniana, Lemna minor, Cucumis sativus, Oryza sativa, Cucurbita spp., Zea mays e Raphanus sativus. 


\section{Material e Métodos}

As plantas aquáticas Egeria najas, Egeria densa, Hydrilla verticillata, Azolla caroliniana, Lemna minor e Wolffia brasiliensis e o caramujo Pomacea canaliculata foram cultivados/criados no setor de cultivo de organismos aquáticos do Laboratório de Ecotoxicologia e Eficácia de Agrotóxicos (LEEA).

Em bandejas de germinação contendo substrato orgânico, foram semeados pepino (Cucumis sativus), milho (Zea mays), abóbora (Cucurbita spp.), rabanete (Raphanus sativus), soja (Glycine max) e arroz (Oryza sativa), em estufa de vegetação. O produto testado foi o sulfentrazone (CAS n $\left.{ }^{\circ} 122836-35-5\right)$ na formulação Boral $^{\circledR}$ com $500 \mathrm{~g}$ ia $\mathrm{L}^{-1}$.

\section{Ensaios de toxicidade aguda para plantas aquáticas}

As plantas aquáticas foram aclimatadas em recipientes de vidro contendo meio de cultivo Hoagland e água $(1: 1 ; \mathrm{v} / \mathrm{v})$, para a aclimatação em sala de bioensaio por 48 horas, em temperatura de $25,0 \pm 2,0{ }^{\circ} \mathrm{C}$, fotoperíodo de 12 horas de luz e 1.000 lux. Após esse período, as plantas com aspecto saudável foram selecionadas e desinfetadas em solução de hipoclorito de sódio a $2 \%$ para L. minor e $3 \%$ para A. caroliniana e água destilada; já $W$. brasiliensis não foi desinfetada. Em seguida, foram transferidos cinco exemplares de $A$. caroliniana e quatro de $L$. minor com três frondes para recipientes-teste contendo $50 \mathrm{~mL}$ de Hoagland, por 24 horas. Por causa do tamanho diminuto da $W$. brasiliensis, foi amostrada uma superfície correspondente a uma área de $19 \mathrm{~mm}$, com o auxílio de um ducto plástico com pressão negativa realizada por uma seringa e um êmbolo.

Para a avaliação de sensibilidade e sanidade, as plantas foram submetidas à exposição à substância referência, que é o cloreto de sódio (99\%), nas concentrações de $0,5,1,12,2,53,5,69,12,81 \mathrm{e}$ $28,83 \mathrm{mg} \mathrm{L}^{-1}$ para $A$. caroliniana e $W$. brasiliensis e de $0,2,0,52,0,91,1,6,2,81$ e $4,92 \mathrm{mg} \mathrm{L}^{-1}$ para L. minor. Os valores das concentrações de inibição $50 \%$ de $\mathrm{NaCl}$ foram de $4,92 \mathrm{mg} \mathrm{L}^{-1}$ para A. caroliniana, de $4,42 \mathrm{mg} \mathrm{L}^{-1}$ para $W$. brasiliensis e de $0,92 \mathrm{mg} \mathrm{L}^{-1}$ para L. minor (Tabela 1).

Após esse período, para L. minor e A. caroliniana foram realizadas as aplicações das concentrações de $0,1,1,0,3,5,11,2$ e $36,5 \mathrm{mg} \mathrm{L}^{-1}$ de sulfentrazone e um controle, todos com três réplicas. Para a W. brasiliensis, foram aplicadas as concentrações de $0,1,1,0,3,5,11,2,36,5$ e $118,0 \mathrm{mg} \mathrm{L}^{-1}$. As avaliações foram realizadas em 3, 5 e 7 dias após a aplicação (número de frondes com clorose e necrose) para $L$. minor, de acordo com Organization for Economic Co-operation and Development (2002), e a porcentagem de danos às colônias de plantas para $A$. caroliniana, de acordo com Silva et al. (2012).

\section{Ensaio de toxicidade para o caramujo (Pomacea canaliculata)}

Para os ensaios de toxicidade aguda, foram utilizados caramujos com peso médio de 2,30 $\pm 0,3 \mathrm{~g}$, aclimatados durante 10 dias em sala de bioensaio com temperatura média de $26 \pm 2{ }^{\circ} \mathrm{C}$ e fotoperíodo de 12 horas, em caixa com capacidade de 60 litros com aeração contínua e alimentação à vontade diária com plantas aquáticas submersas $E$. najas e E. densa.

Os ensaios foram conduzidos no sistema estático, sem substituição e sem sifonagem de água, com período de exposição de 48 horas e os animais mantidos sem alimentação. Os parâmetros físico-químicos iniciais da água no teste foram: condutividade elétrica, $124,96 \pm 1,51 \mu \mathrm{s} \mathrm{cm}^{-1}$; oxigênio dissolvido, $2,48 \pm 0,01 \mathrm{mg} \mathrm{L}^{-1} ; \mathrm{pH}$, $8,23 \pm 0,04$; e temperatura, $25,70 \pm 0,04{ }^{\circ} \mathrm{C}$.

Para a avaliação de sensibilidade e sanidade, os caramujos foram submetidos à exposição à substância referência, que é o cloreto de potássio (99\%), nas concentrações de $0,09,0,8,1,4,2,45$,

Tabela 1. Sensibilidade dos organismos bioindicadores aquáticos a substâncias referências.

\begin{tabular}{|c|c|c|c|}
\hline Organismo-teste & $\begin{array}{c}\text { Intervalo de confiança } \\
\text { de } 95 \% \text { inferior }\end{array}$ & $\operatorname{CL} / \operatorname{CE50}\left(\mathrm{mg} \mathrm{L}^{-1}\right)$ & $\begin{array}{c}\text { Intervalo de confiança } \\
\text { de } 95 \% \text { superior }\end{array}$ \\
\hline P. canaliculata & 0,88 & 1,08 & 1,32 \\
\hline L. minor & 0,86 & 0,92 & 0,98 \\
\hline A. caroliniana & 3,99 & 4,92 & 6,08 \\
\hline W. brasiliensis & 3,42 & 4,42 & 4,85 \\
\hline
\end{tabular}


4,2 e 7,5 $\mathrm{mg} \mathrm{L}^{-1}$ e um controle. Os resultados de sensibilidade estão apresentados na Tabela 1.

Nos ensaios definitivos, foram utilizadas seis concentrações e um controle, sendo $0,1,1,0,3,5$, $11,2,36,5$ e $118,0 \mathrm{mg} \mathrm{L}^{-1}$, com três repetições. A avaliação da mortalidade foi realizada em $24 \mathrm{e}$ 48 horas, com a retirada dos caramujos mortos (imóveis e com os opérculos abertos) dos recipientes. Também foi realizada a análise da qualidade de água ( $\mathrm{pH}$, condutividade elétrica, oxigênio dissolvido e temperatura).

\section{Ensaio de toxicidade aguda para plantas- teste terrestres}

Os ensaios de toxicidade aguda (CL50;14d) foram realizados após as plantas-teste emitirem as três primeiras folhas verdadeiras. Estas foram transplantadas em recipientes plásticos contendo $300 \mathrm{~g}$ de areia fina $(2,0 \mu \mathrm{m})$ e lavadas.

Os ensaios foram conduzidos em sala de bioensaio com temperatura de $25,0 \pm 2,0{ }^{\circ} \mathrm{C}$ e fotoperíodo de 12 horas de luz. Os testes foram realizados nas concentrações de $0,1,1,0,3,5,11,2 \mathrm{e}$ $36,5 \mathrm{mg} \mathrm{kg}^{-1}$. Para as plantas de pepino (C. sativus), foram testadas as concentrações de $0,01,0,06$, $0,1,0,7 \mathrm{e} 3,5 \mathrm{mg} \mathrm{kg}^{-1}$. Os testes foram realizados com cinco réplicas por concentração, incluindo o controle, contendo duas plantas cada.

Para a diluição de cada concentração-teste, foram utilizados como solução de diluição $70,0 \mathrm{~mL}$ de água destilada, equivalente à máxima umidade da areia, previamente aferida. A cada 24 horas foram repostos $30,0 \mathrm{~mL}$ de água em cada unidade experimental para manter a umidade da areia para que não ocorresse limitação no desenvolvimento das plantas em razão do estresse hídrico.

Ao final do período de 14 dias, as plantas foram retiradas das parcelas experimentais para mensurar o desenvolvimento da parte aérea $(\mathrm{cm})$, a emissão de raízes $(\mathrm{cm})$ e da produção de biomassa fresca $(\mathrm{g})$ e a produção de biomassa seca (g) com o peso obtido em balança analítica. A avaliação da mortalidade foi realizada de acordo com Nascimento \& Yamashita (2009).

\section{Avaliação de efeito de subdoses nas plantas aquáticas submersas}

Para a determinação do efeito de subdoses, os ensaios foram realizados em sala de bioensaio com temperatura de $25,0 \pm 2,0{ }^{\circ} \mathrm{C}$, fotoperíodo de 12 horas de luz e 1.000 lux. Para tanto, foram selecionados e coletados fragmentos apicais de $7,0 \mathrm{~cm}$ das plantas aquáticas E. najas, E. densa e $H$. verticillata de bom aspecto sanitário (sem sinal de clorose ou necrose). Estes foram transferidos para tubos de ensaio com capacidade de $100 \mathrm{~mL}$, contendo $70 \mathrm{~mL}$ de água, por 24 horas para aclimatação. Nos ensaios definitivos, as concentrações utilizadas foram de $0,01,0,05,0,1$, $1,0,2,5$ e $5,0 \mathrm{mg} \mathrm{L}^{-1}$, também com um controle com dez repetições cada.

O período de exposição das plantas aquáticas ao herbicida foi de 14 dias. Ao final desse período experimental, foram realizadas as avaliações da emissão de raízes e crescimento total $(\mathrm{cm})$, conforme Peres et al. (2017).

\section{Análise estatística}

A concentração letal (CL50;7d) para as plantas aquáticas e (CL50;14d) para as plantas terrestres e os resultados da relação concentração-resposta (mortalidade) foram submetidos à análise regressão linear, e os valores de CE50;48h foram calculados por meio do software Trimmed SpearmanKarber (Hamilton et al., 1977), e a classificação ecotoxicológica foi realizada de acordo com a classificação da United States Environmental Protection Agency (2017). Os dados, o comprimento de parte aérea e o crescimento radicular obtidos foram submetidos à análise de variância (ANOVA), e as médias foram comparadas por Tukey a $5 \%$.

\section{Resultados e Discussão}

Os resultados de sensibilidade para o cloreto de sódio $(\mathrm{NaCl})$ e o cloreto de potássio $(\mathrm{KCl})$ dos organismos aquáticos estão apresentados na Tabela 1.

\section{Toxicidade aguda do Sulfentrazone}

Para A. caroliniana, a concentração letal $50 \%$ (CL50;7d) foi $>118,0 \mathrm{mg} \mathrm{L}^{-1}$, sendo esse valor superior à concentração limite para ensaios de toxicidade aguda para peixes, segundo a Organization for Economic Co-operation and Development (2009), que recomenda a maior concentração testada de $100,0 \mathrm{mg} \mathrm{L}^{-1}$ para o produto químico ser considerado praticamente não tóxico. Para esse bioindicador, o sulfentrazone foi menos tóxico que o oxyfluorfen com CL50;7d de $80,0 \mathrm{mg} \mathrm{L}^{-1}$ e o mesmo mecanismo de ação que o sulfentrazone, 
sendo classificado como pouco tóxico (Silva et al., 2012). O sulfentrazone também foi menos tóxico que o imazapyr com CL50;7d de 18,98 $\mathrm{mg} \mathrm{L}^{-1}$ também para A. caroliniana (Cruz et al., 2015) e toxicidade similar aos herbicidas atrazine, bentazon + imazamox e clomazone para esse bioindicador (Della Vechia et al., 2016).

A relação concentração-porcentagem de inibição do crescimento/mortalidade pode ser observada nas concentrações de $0,1,1,07,3,5$, $11,2,36,5$ e $118,0 \mathrm{mg} \mathrm{L}^{-1}$, com porcentagem de inibição no crescimento de 5, 10, 13, 20, 25 e 30\%, respectivamente. Ainda, nenhuma concentração apresentou necrose total.

Para $W$. brasiliensis, a concentração letal 50\% (CL50;7d) foi $>118,0 \mathrm{mg} \mathrm{L}^{-1}$ (Tabela 2), sendo considerada praticamente não tóxica. A relação concentração-porcentagem de inibição do crescimento/ mortalidade pode ser observada nas concentrações de $0,1,1,07,3,5,11,2,36,5$ e $118,0 \mathrm{mg} \mathrm{L}^{-1}$, com porcentagem de inibição no crescimento de 0,10 , $15,20,40$ e $50 \%$, respectivamente. A toxicidade aguda do sulfentrazone foi similar a atrazine, 2,4-D, saflufenacil, imazamox, penoxsulam e metsulfuronmethyl. Não foi possível estimar a concentração letal $50 \%$ por causa da ausência de ocorrência de mortalidade padronizada em relação ao aumento das concentrações testadas; assim, a CL50;7d para a $W$. brasiliensis para esses herbicidas foi considerada $>118,0 \mathrm{mg} \mathrm{L}^{-1}$ (Pereira et al., 2019).

Para L. minor, a concentração letal 50\% (CL50;7d) foi de $0,80 \mathrm{mg} \mathrm{L}^{-1}$, com limite superior de $0,97 \mathrm{mg} \mathrm{L}^{-1} \mathrm{e}$ limite inferior de $0,65 \mathrm{mg} \mathrm{L}^{-1}$ (Tabela 2), sendo classificada como altamente tóxica (United States Environmental Protection Agency, 2017), com equação linear que representa a relação concentração-mortalidade de $\mathrm{y}=7,61 \mathrm{x}-7,45$, com correlação de $\mathrm{R}^{2}=0,86$. Assim, ocorreu redução de crescimento dos frondes em 0,1 e $1,07 \mathrm{mg}$ $\mathrm{L}^{-1}$ de $0 \%$; em 3,50 $\mathrm{mg} \mathrm{L}^{-1}$, de 63,16\%; em 11,20 mg $\mathrm{L}^{-1}$, de $89,47 \%$; e em $36,5 \mathrm{mg} \mathrm{L}^{-1}$, de $91,23 \%$. O sulfentrazone foi mais tóxico para a L. minor do que imazapyr na CL50;7d de 21,10 $\mathrm{mg} \mathrm{L}^{-1}$ (Pereira et al., 2019) e similar ao imazapyr na CL50;7d de 1,06 mg $\mathrm{L}^{-1}$ para a L. minor (Cruz et al., 2015). Esse herbicida foi mais tóxico que os herbicidas atrazine, bentazon + imazamox e clomazone, também para a L. minor, com CL50;7d de 5,27,31,58 e 10,28 mg $\mathrm{L}^{-1}$, respectivamente (Della Vechia et al., 2016). Para $P$. canaliculata, a CE50;48h foi $>$ $118,0 \mathrm{mg} \mathrm{L}^{-1}$ (Tabela 2), sendo considerada praticamente não tóxica. Esse organismo também não foi sensível às duas formulações testadas do inseticida lambda-cialotrina (Leonel et al., 2016) e ao antibiótico florfenicol (Florêncio et al., 2014). Porém, o caramujo apresentou excelente resposta ao inseticida toltrazuril e sua mistura com o antibiótico enrofloxacina com CE50;48h de 7,59 e $12,34 \mathrm{mg} \mathrm{L}^{-1}$ (Carraschi et al., 2016) e ao imazapyr com CE50;48h de 5,89 $\mathrm{mg} \mathrm{L}^{-1}$ (Cruz et al., 2015).

A toxicidade aguda (CL50;14d) do sulfentrazone para o pepino (C. sativus) foi de $0,24 \mathrm{mg} \mathrm{kg}^{-1}$ de areia (Tabela 3), sendo a planta mais sensível ao herbicida, enquanto a CL50;14d foi de 4,52 $\mathrm{mg} \mathrm{kg}^{-1}$ para a abóbora (Cucurbita spp.), similar à toxicidade (IC50;7d) do imazethapyr com 0,61 a $3,22 \mathrm{mg}$ $\mathrm{kg}^{-1}$ (solo em pH 5,5 a 8,5) e de 2,4-D com 1,87 mg $\mathrm{kg}^{-1}$ (solo em pH 5,5) para o arroz (O. sativa) (Liu et al., 2018).

As espécies de plantas-teste mais sensíveis ao herbicida foram pepino (C. sativus) $>\operatorname{soja}(G$. max $)$ $>$ milho $(Z$. mays $)>$ rabanete $($ R. sativus $)>$ arroz

Tabela 2. Toxicidade aguda do sulfentrazone para organismos aquáticos e terrestres.

\begin{tabular}{cccc}
\hline Organismo-teste & Limite superior & $\begin{array}{c}\mathbf{C L} / \mathbf{C E 5 0} \\
(\mathbf{m g ~ L} \mathbf{- 1} \mathbf{m g ~ k g} \mathbf{~} \mathbf{- 1}\end{array}$ & Limite inferior \\
\hline P. canaliculata & - & $>118,0$ & - \\
L. minor & 0,65 & 0,80 & 0,97 \\
A. caroliniana & - & $>118,0$ & - \\
W. brasiliensis & - & $>118,0$ & - \\
C. sativus & 0,32 & 0,24 & 0,19 \\
Z. mays & 1,67 & 1,20 & 0,86 \\
Cucurbita spp & 5,82 & 4,52 & 3,51 \\
R. sativus & 1,91 & 1,40 & 1,03 \\
G. max & 1,96 & 1,16 & 0,69 \\
O. sativa & 3,61 & 1,67 & 0,77 \\
\hline
\end{tabular}

$(-)=$ Sem limite de confiança; CL/CE50 = Concentração letal 50\% (CL 50). 
(O. sativa) > abóbora (Cucurbita spp.) de acordo com a toxicidade aguda e mortalidade (Tabela 2). A relação de concentração-mortalidade das plantasteste está apresentada na Tabela 3, sendo que para C. sativus ocorreram $100 \%$ de mortalidade na concentração de $5,0 \mathrm{mg} \mathrm{kg}^{-1}$, enquanto para $Z$. mays, $R$. sativus e $O$. sativa, na concentração de $36,50 \mathrm{mg} \mathrm{kg}^{-1}$.

Também foi observada redução do comprimento médio total das plantas e foram avaliadas a porcentagem média de redução (Tabela 4) e a porcentagem de redução de massa fresca e seca (Tabela 5), além de terem sido feitos os cálculos da CL50;14d (Tabela 2). O pepino (C. sativus) foi mais sensível em relação às outras plantasteste, assim como o próprio C. sativus, exposto às concentrações de $1,0 \mathrm{mg} \mathrm{L}^{-1}$ dos herbicidas triclopyr e 2,4-D, com resposta sensível e 100\% de mortalidade no $14^{\circ}$ dia após a aplicação (Bastos, 2015).

\section{Efeito de subdose}

Para a $E$. densa, o crescimento médio do fragmento apical em relação ao controle ocorreu nas concentrações 0,01 e $0,05 \mathrm{mg} \mathrm{L}^{-1}$, que se diferiram significativamente em relação às demais concentrações. Nas outras ocorreu redução do comprimento total médio do fragmento apical em relação ao controle (Tabela 6). As concentrações testadas indicam efeito do herbicida a partir de $1,0 \mathrm{mg} \mathrm{L}^{-1}$, porém ocorreram 26,6 e $17 \%$ de estímulo de crescimento dos fragmentos apicais

Tabela 3. Porcentagem (\%) de mortalidade das plantas-teste durante os ensaios de toxicidade aguda com sulfentrazone.

\begin{tabular}{ccccccc}
\hline $\begin{array}{c}\text { Tratamentos } \\
\left(\mathbf{m g ~ L}^{-1}\right)\end{array}$ & C. sativus & Z. mays & $\begin{array}{c}\text { Cucurbita } \\
\text { spp. }\end{array}$ & R. sativus & G. max & O. sativa \\
\hline $\mathbf{0 , 0 0}$ & 0,0 & 0,0 & 0,0 & 0,0 & 0,0 & 0,0 \\
$\mathbf{0 , 0 1}$ & 10,0 & - & - & - & - & - \\
$\mathbf{0 , 0 6}$ & 15,0 & - & - & - & - & - \\
$\mathbf{0 , 1 0}$ & 35,0 & 18,0 & 13,0 & 19,0 & 30,0 & 35,0 \\
$\mathbf{0 , 7 0}$ & - & - & - & - & - & - \\
$\mathbf{1 , 0 7}$ & - & 39,0 & 14,0 & 30,0 & 40,0 & 40,0 \\
$\mathbf{3 , 5 0}$ & 100,0 & 74,0 & 32,0 & 82,0 & 75,0 & 60,0 \\
$\mathbf{5 , 0 0}$ & 100,0 & - & - & - & - & - \\
$\mathbf{1 1 , 1 6}$ & - & 95,0 & 87,0 & 99,0 & 90,0 & 70,0 \\
$\mathbf{3 6 , 5 0}$ & - & 100,0 & 96,0 & 100,0 & 98,0 & 100,0 \\
$\mathbf{1 1 8 , 0 0}$ & - & 100,0 & 100,0 & 100,0 & 100,0 & 100,0 \\
\hline
\end{tabular}

$(-)=$ Concentração não avaliada neste ensaio.

Tabela 4. Porcentagem (\%) de redução do tamanho médio total e sinais/mortalidade das plantas expostas ao herbicida sulfentrazone.

\begin{tabular}{|c|c|c|c|c|c|c|c|c|c|c|c|c|}
\hline \multirow{2}{*}{$\begin{array}{c}\text { Trat. } \\
\text { mg L }^{-1}\end{array}$} & \multicolumn{2}{|c|}{ C. sativus } & \multicolumn{2}{|c|}{ Z. mays } & \multicolumn{2}{|c|}{ C. spp. } & \multicolumn{2}{|c|}{ R. sativus } & \multicolumn{2}{|c|}{ G. $\max$} & \multicolumn{2}{|c|}{ O. sativa } \\
\hline & $\begin{array}{c}\% \\
\text { R.C }\end{array}$ & $\begin{array}{c}\% \\
\text { S/M }\end{array}$ & $\begin{array}{c}\% \\
\text { R.C }\end{array}$ & $\begin{array}{c}\% \\
\text { S/M }\end{array}$ & $\begin{array}{c}\% \\
\text { R.C }\end{array}$ & $\begin{array}{c}\% \\
\text { S/M }\end{array}$ & $\begin{array}{c}\% \\
\text { R.C }\end{array}$ & $\begin{array}{c}\% \\
\text { S/M }\end{array}$ & $\begin{array}{c}\% \\
\text { R.C }\end{array}$ & $\begin{array}{c}\% \\
\text { S/M }\end{array}$ & $\begin{array}{c}\% \% \\
\text { R.C }\end{array}$ & $\begin{array}{c}\% \\
\text { S/M }\end{array}$ \\
\hline 0,00 & 0 & 0 & 0 & 0 & 0 & 0 & 0 & 0 & 0 & 0 & 0 & 0 \\
\hline 0,01 & 2,94 & 10 & - & - & - & - & - & - & - & - & - & - \\
\hline 0,06 & 8,13 & 15 & - & - & - & - & - & - & - & - & - & - \\
\hline 0,10 & 25,43 & 35 & 9,72 & 18 & 12,41 & 13 & 12,75 & 19 & 59,60 & 30 & 18,52 & 35 \\
\hline 0,70 & 65,22 & 70 & - & - & - & - & - & - & - & - & - & - \\
\hline 1,07 & - & - & 29,17 & 39 & 15,17 & 14 & 20,80 & 30 & 63,94 & 40 & 40 & 40 \\
\hline 3,50 & 100 & 100 & 75,27 & 74 & 15,86 & 32 & 52,01 & 82 & 65,21 & 75 & 60 & 60 \\
\hline 5,00 & 100 & 100 & - & - & - & - & - & - & - & - & - & - \\
\hline 11,16 & - & - & 95,19 & 95 & 71,03 & 87 & 89,93 & 99 & 65,21 & 90 & 70 & 70 \\
\hline 36,50 & - & - & 100 & 100 & 83,44 & 96 & 100 & 100 & 90,58 & 98 & 100 & 100 \\
\hline 118,00 & - & - & 100 & 100 & 100 & 100 & 100 & 100 & 100 & 100 & 100 & 100 \\
\hline
\end{tabular}

\% R.C. Redução de crescimento; \% S/M. Sinais/Mortalidade; (-) Concentração não presente neste ensaio. 
Tabela 5. Porcentagem (\%) de redução média de massa fresca e seca total das plantas expostas ao herbicida sulfentrazone.

\begin{tabular}{|c|c|c|c|c|c|c|c|c|c|c|c|c|}
\hline \multirow{2}{*}{$\begin{array}{c}\text { Trat. } \\
\left(\mathrm{mg} \mathrm{L}^{-1}\right)\end{array}$} & \multicolumn{2}{|c|}{ C. sativus } & \multicolumn{2}{|c|}{ Z. mays } & \multicolumn{2}{|c|}{ C. spp } & \multicolumn{2}{|c|}{ R. sativus } & \multicolumn{2}{|c|}{ G. $\max$} & \multicolumn{2}{|c|}{ O. sativa } \\
\hline & M. F. & M.S. & M. F. & M.S. & M. F. & M.S. & M. F. & M.S. & M. F. & M.S. & M. F. & M.S. \\
\hline 0,00 & 0 & 0 & 0 & 0 & 0 & 0 & 0 & 0 & 0 & 0 & 0 & \\
\hline 0,01 & 24,6 & 23,3 & - & - & - & - & - & - & - & - & - & - \\
\hline 0,06 & 31,8 & 45,9 & - & - & - & - & - & - & - & - & - & - \\
\hline 0,10 & 45,0 & 1,0 & 23,3 & 22,4 & 10,9 & 3,9 & 11,0 & 44,4 & 30,9 & 55,7 & 3,6 & 35,1 \\
\hline 0,70 & 70,1 & 79 & - & - & - & - & - & - & - & - & - & - \\
\hline 1,07 & - & - & 37,5 & 29,5 & 13,62 & 7,89 & 19,21 & 44,44 & 47,83 & 42,9 & 23,4 & 22,2 \\
\hline 3,50 & 100 & 100 & 72,3 & 72,4 & 13,13 & 6,57 & 60,52 & 55,55 & 56,42 & 71,9 & 61,5 & 74,0 \\
\hline 5,00 & 100 & 100 & - & - & - & - & - & - & - & - & - & - \\
\hline 11,16 & - & - & 93,4 & 93,2 & 73,80 & 69,73 & 94,74 & 94,44 & 65,07 & 78,9 & 63,06 & 79,6 \\
\hline 36,50 & - & - & 100 & 100 & 82,96 & 84,21 & 100 & 100 & 92 & 95,1 & 100 & 100 \\
\hline 118,00 & - & - & 100 & 100 & 100 & 100 & 100 & 100 & 100 & 100 & 100 & 100 \\
\hline
\end{tabular}

- Concentração não presente neste ensaio.

Tabela 6. Efeitos no crescimento dos fragmentos apicais e de raiz de E. densa exposta a subdoses de sulfentrazone.

\begin{tabular}{cccccrr}
\hline $\begin{array}{c}\text { Conc. } \\
\left(\mathbf{m g ~ L} \mathbf{~}^{-1}\right)\end{array}$ & $\begin{array}{c}\text { Cresc. apical } \\
(\mathbf{c m})\end{array}$ & \% cresc. & \% redução & $\begin{array}{r}\text { Cresc. de } \\
\text { raiz }(\mathbf{c m})\end{array}$ & \% cresc. & \% redução \\
\hline $\mathbf{0 , 0 0}$ & $11,4 \mathrm{~b}$ & 100,0 & 0,0 & $\mathbf{4 , 1 a}$ & $\mathbf{1 0 0 , 0}$ & $\mathbf{0 , 0}$ \\
$\mathbf{0 , 0 1}$ & $14,5 \mathrm{a}$ & 126,6 & $-26,6$ & $\mathbf{2 , 8 a b}$ & $\mathbf{6 7 , 4}$ & $\mathbf{3 2 , 5}$ \\
$\mathbf{0 , 0 5}$ & $13,4 \mathrm{ab}$ & 117,0 & $-17,0$ & $\mathbf{2 , 1 a b}$ & $\mathbf{5 0 , 6}$ & $\mathbf{4 9 , 3}$ \\
$\mathbf{0 , 1 0}$ & $11,2 \mathrm{~b}$ & 97,8 & 2,1 & $\mathbf{0 , 5 a b}$ & $\mathbf{1 3 , 2}$ & $\mathbf{8 6 , 7}$ \\
$\mathbf{1 , 0 0}$ & $7,8 \mathrm{c}$ & 68,1 & 31,8 & $\mathbf{0 , 0 b}$ & $\mathbf{- 1 0 0 , 0}$ & $\mathbf{1 0 0 , 0}$ \\
$\mathbf{2 , 5 0}$ & $7,9 \mathrm{c}$ & 69,4 & 30,5 & $\mathbf{0 , 0 b}$ & $\mathbf{- 1 0 0 , 0}$ & $\mathbf{1 0 0 , 0}$ \\
$\mathbf{5 , 0 0}$ & $7,8 \mathrm{c}$ & 68,5 & 31,4 & $\mathbf{0 , 0 b}$ & $\mathbf{- 1 0 0 , 0}$ & $\mathbf{1 0 0 , 0}$ \\
$\mathbf{D M S ~ 5 \%}$ & 2,58 & - & - & 4,48 & - & - \\
$\mathbf{F} *$ & $21,62 * *$ & - & - & $3,18 * *$ & - & - \\
$\mathbf{C V}$ & 17,88 & - & - & 215,29 & - & - \\
\hline
\end{tabular}

Letras minúsculas diferentes na coluna indicam diferença significativa pelo teste de Tukey a 5\%; (-) indica não calculado para este item.

em 0,01 e $0,05 \mathrm{mg} \mathrm{L}^{-1}$, respectivamente, similares ao efeito de 1,0 a $11,2 \mathrm{mg} \mathrm{L}^{-1}$ de 2,4-D para $E$. densa com estímulo de $90 \%$ de crescimento (Peres et al., 2017) e de $30,0 \mu \mathrm{g} \mathrm{L}^{-1}$ de 2,4-D para Ranunculus aquatilis (Belgers et al., 2007), indicando efeito de hormese do sulfentrazone para $E$. densa.

Para a raiz, foi observada a redução do tamanho médio em relação ao controle nas concentrações 0,01 , 0,05 e $0,10 \mathrm{mg} \mathrm{L}^{-1}$; nas demais concentrações testadas, a inibição do crescimento radicular foi total. Segundo Carvalho (2013), isso ocorre em casos de sintomas da intoxicação por inibidores de ALS ou AHAS, que têm como sinal a inibição do crescimento radicular da planta.

Para E. najas, houve maior crescimento dos fragmentos apicais em relação ao controle nas concentrações $0,01,0,05,0,1$ e $2,5 \mathrm{mg} \mathrm{L}^{-1}$, no entanto sem diferença entre os tratamentos. Também ocorreu diminuição do emissão de raiz em comparação ao controle em todas as concentrações aplicadas (Tabela 7), o que demonstra possível efeito do herbicida para a E. najas do sulfentrazone, similar ao descrito com clomazone para essa planta aquática (Peres et al., 2017).

Para $H$. verticillata nas concentrações 0,01 , 0,05 e $0,1 \mathrm{mg} \mathrm{L}^{-1}$, ocorreu o crescimento médio dos fragmentos apicais similar ao controle. Nas demais concentrações, houve a diminuição do comprimento médio total em relação ao controle. Para o comprimento médio de raiz em comparação ao controle, ocorreu crescimento nas concentrações 0,5 e $2,5 \mathrm{mg} \mathrm{L}^{-1}$, enquanto nas demais concentrações e no controle não houve emissão de raiz (Tabela 8). Peres et al. 
Tabela 7. Efeitos no crescimento dos fragmentos apicais e de raiz de E. najas exposta a subdoses de sulfentrazone.

\begin{tabular}{ccccccr}
\hline $\begin{array}{c}\text { Conc. } \\
\left(\mathbf{m g ~ L} \mathbf{~}^{-1}\right)\end{array}$ & $\begin{array}{c}\text { Cresc. apical } \\
(\mathbf{c m})\end{array}$ & \% cresc. & \% redução & $\begin{array}{c}\text { Cresc. de } \\
\text { raiz }(\mathbf{c m})\end{array}$ & \% cresc. & \% redução \\
\hline $\mathbf{0 , 0 0}$ & $8,0 \mathrm{a}$ & 100,0 & 0,0 & $7,5 \mathrm{a}$ & 100,0 & 0,0 \\
$\mathbf{0 , 0 1}$ & $9,6 \mathrm{a}$ & 120,1 & $-20,1$ & $2,7 \mathrm{bc}$ & 63,6 & 36,3 \\
$\mathbf{0 , 0 5}$ & $9,7 \mathrm{a}$ & 120,9 & $-20,9$ & $5,4 \mathrm{ab}$ & 28,0 & 71,9 \\
$\mathbf{0 , 1 0}$ & $10,5 \mathrm{a}$ & 131,0 & $-31,0$ & $1,4 \mathrm{c}$ & 80,9 & 19,0 \\
$\mathbf{1 , 0 0}$ & $8,5 \mathrm{a}$ & 106,2 & $-6,9$ & $0,0 \mathrm{c}$ & $-100,0$ & 100,0 \\
$\mathbf{2 , 5 0}$ & $8,6 \mathrm{a}$ & 106,9 & $-6,9$ & $0,0 \mathrm{c}$ & $-100,0$ & 100,0 \\
$\mathbf{5 , 0 0}$ & $7,5 \mathrm{a}$ & 93,8 & 6,2 & $0,0 \mathrm{c}$ & $-100,0$ & 100,0 \\
$\mathbf{D M S ~ 5 \%}$ & 3,59 & - & - & 3,48 & - & - \\
$\mathbf{F} *$ & $1,65 \mathrm{NS}$ & - & - & $14,04 * *$ & - & - \\
$\mathbf{C V}$ & 26,04 & - & - & 92,38 & - & - \\
\hline
\end{tabular}

Letras minúsculas diferentes na coluna indicam diferença significativa pelo teste de Tukey a 5\%; (-) indica não calculado para este item.

Tabela 8. Efeitos no crescimento dos fragmentos apicais e de raiz de $H$. verticillata exposta a subdoses de sulfentrazone.

\begin{tabular}{ccccccc}
\hline $\begin{array}{c}\text { Conc. } \\
\left.(\mathbf{m g ~ L})^{-1}\right)\end{array}$ & $\begin{array}{c}\text { Cresc. apical } \\
(\mathbf{c m})\end{array}$ & \% cresc. & \% redução & $\begin{array}{c}\text { Cresc. de } \\
\text { raiz }(\mathbf{c m})\end{array}$ & \% cresc. & \% redução \\
\hline $\mathbf{0 , 0 0}$ & $\mathbf{1 0 , 2 a}$ & $\mathbf{1 0 0 , 0}$ & $\mathbf{0 , 0}$ & $\mathbf{0 , 2 a}$ & $\mathbf{1 0 0 , 0}$ & $\mathbf{0 , 0}$ \\
$\mathbf{0 , 0 1}$ & $\mathbf{1 0 , 2 a}$ & $\mathbf{1 0 0 , 4}$ & $\mathbf{- 0 , 4}$ & $\mathbf{0 , 0 a}$ & $\mathbf{0 , 0}$ & $\mathbf{1 0 0 , 0}$ \\
$\mathbf{0 , 0 5}$ & $\mathbf{1 0 , 2 ^ { \mathbf { a } }}$ & $\mathbf{1 0 0 , 4}$ & $\mathbf{- 0 , 4}$ & $\mathbf{0 , 3 a}$ & $\mathbf{1 4 0 , 0}$ & $\mathbf{- 4 0 , 0}$ \\
$\mathbf{0 , 1 0}$ & $\mathbf{1 0 , 9 a}$ & $\mathbf{1 0 7 , 3}$ & $\mathbf{7 , 3}$ & $\mathbf{0 , 0 a}$ & $\mathbf{0 , 0}$ & $\mathbf{1 0 0 , 0}$ \\
$\mathbf{1 , 0 0}$ & $\mathbf{9 , 0 a}$ & $\mathbf{8 8 , 2}$ & $\mathbf{1 1 , 7}$ & $\mathbf{0 , 0 a}$ & $\mathbf{0 , 0}$ & 100,0 \\
$\mathbf{2 , 5 0}$ & $\mathbf{9 , 2 a}$ & $\mathbf{9 0 , 1}$ & $\mathbf{9 , 8}$ & $\mathbf{0 , 6 a}$ & $\mathbf{2 4 0 , 0}$ & $\mathbf{- 1 4 0 , 0}$ \\
$\mathbf{5 , 0 0}$ & $\mathbf{8 , 0 a}$ & $\mathbf{8 6 , 2}$ & $\mathbf{1 3 , 7}$ & $\mathbf{0 , 0 a}$ & 100,0 & 0,0 \\
$\mathbf{D M S} \mathbf{5} \%$ & 2,56 & - & - & 1,072 & - & - \\
F $^{*}$ & $1,83 \mathrm{NS}$ & - & - & $0,91 \mathrm{NS}$ & - & - \\
$\mathbf{C V}$ & 19,17 & - & - & 459,25 & - & - \\
\hline
\end{tabular}

Letras minúsculas diferentes na coluna indicam diferença significativa pelo teste de Tukey a 5\%; (-) indica não calculado para este item.

(2017) relataram crescimento significativo para E. najas exposta ao herbicida 2,4-D nas concentrações de 1,0 a $11,2 \mathrm{mg} \mathrm{L}^{-1}$. Belgers et al. (2007) também observaram efeito de subdose de 2,4-D em oito de nove espécies testadas de plantas aquáticas, sendo que, para Potamogeton lucens, a concentração de maior crescimento foi de $10,0 \mu \mathrm{g} \mathrm{L}^{-1}$; para Ranunculus circinatus, Elodea nuttallii, Ceratophyllum demersum e Ranunculus peltatus, de $30,0 \mu \mathrm{g} \mathrm{L}^{-1}$; para Potamogeton crispus e Ranunculus aquatilis, de $100,0 \mu \mathrm{g} \mathrm{L}^{-1}$; e para Lemna trisulca, de 300,0 $\mu \mathrm{g} \mathrm{L}^{-1}$.

\section{Conclusões}

Os organismos-teste L. minor (planta aquática) e C. sativus (planta terrestre) apresentaram excelente resposta de sensibilidade ao sulfentrazone, indicando potencial para bioindicação, no entanto todas as plantas terrestres mostraram potencial para o biomonitoramento.

Para as plantas aquáticas, ocorreu estímulo de crescimento do fragmento apical para a planta aquática $E$. densa, o que indica possível efeito de hormese desse herbicida, sendo necessário cuidado com essa molécula para não atingir o ambiente aquático.

\section{Agradecimentos}

Ao programa de PIBIC/CNPq/Unifeb, pela concessão da bolsa de iniciação científica ao primeiro autor.

\section{Referências}

Adger, W. N., Brown, K., \& Conway, D. (2010). Progress in global environmental change. In J. Barnett, L. Lebel, M. New, K. Seto. (Ed.), Global 
Environmental Change (4a ed., pp.547-549). Chang: Elsevier.

Agathokleous, E., Anav, A., Araminiene, V., de Marco, A., Domingos, M., Kitao, M., Koike, T., Manning, W. J., Paoletti, E., Saitanis, C. J., Sicard, P., Vitale, M., Wang, W., Calabrese, E. J. (2019a). Commentary: EPA's proposed expansion of dose-response analysis is a positive step towards improving its ecological risk assessment. In: C. Sonne, \& E. Zeng (Eds.), Environmental Pollution (pp. 566-570) .

Agathokleous, E., \& Calabrese, E. J. (2020). Science of the Total Environment: A global environmental health perspective and optimisation of stress. Science Direct, 704, 135263.

Agathokleous, E., Kitao, M., \& Calabrese, E. J. (2019b). Hormesis: A compelling platform for sophisticated plant science. Trends in Plant Science, 24, 24-37.

Bastos, J. C. H. A. G. (2015). Avaliação da sensibilidade de organismos aquáticos e terrestres ao triclopyr (Tese de doutorado). Universidade Estadual Paulista "Júlio de Mesquita Filho" Faculdade de Ciências Agrárias e Veterinárias Campus de Jaboticabal, Jaboticabal.

Bertoncelli, D. J., Alves, G. A. C., Furlan, F. F., Freiria, G. H., Bazzo, J. H. B., \& Faria, R. T. (2018). Efeito do glifosato no cultivo in vitro de Cattleya nobilior rchb. F. Ceres, 65(2), 165-173.

Belgers, J. D. M., Lieverloo, R. J., Van Der Pas, L. J. T., \& Van Den Brink, P. J. (2007). Effects of the herbicide 2,4-D on the growth of nine aquatic macrophytes. Aquatic Botany, 86(3), 260-268.

Blanco, F. M. G., \& Velini, E. D. (2005). Persistência do herbicida sulfentrazone em solo cultivado com soja e seu efeito em culturas sucedâneas. Planta Daninha, 23(4), 693-700.

Calabrese, E. J., \& Blain, R. B. (2011). The hormesis database: the occurence of hermetic dose responses in the toxicological literature. Regulatory Toxicology and Pharmacology, 61, 73-81.

Carraschi, S. P., Florêncio, T., Cruz, C., \& RanzaniPaiva, M. J. T. (2016). Avaliação ecotoxicológica de associações de fármacos utilizados na aquicultura. Ciência e Cultura, 12(1), 27-36.
Carvalho, L. B. (2013). Herbicidas (1a ed.). Lages: Editado pelo autor.

Cruz, C., Silva, A. F., Shiogiri, N. S., Garlich, N., \& Pitelli, R. A. (2015). Imazapyr herbicide efficacy on floating macrophyte control and ecotoxicology for non-target organisms. Planta Daninha, 33(1), 103-108.

Della Vechia, J. F., Cruz, C., Silva, A. F., CerveiraJR, W. R., \& Garlich, N. (2016). Macropyte bioassay applications for monitoring pesticides inthe aquatic environment. Planta Daninha, 34(3), 597-603.

Fischer, A. P. (2019). Adapting and coping with climate change in temperate forests. Global Environmental Change, 54, 160-171.

Florêncio, T., Carraschi, S. P., Cruz, C., Silva, A. F., Marques, A. M., \& Pitelli, R. A. (2014). Bioindicadores neotropicais de ecotoxicidade e risco ambiental de fármacos de interesse para aquicultura. Boletim do Instituto de Pesca, 40(04), 569-576.

Foloni, L. L., \& Pitelli, R. A. (2005). Avaliação da sensibilidade de diversas espécies de plantas daninhas aquáticas ao carfentrazone-ethyl, em ambiente controlado. Planta Daninha, 23(2), 329-334.

Hamilton, M. A., Russo, R. C., \& Thurston, R. V. (1977). Trimmed spearman - karber method for estimating medical lethal concentrations in toxicity bioassays. Environmental Science \& Technology, 7, 714-719.

Leonel, K. P., Morandi, J. M., Brunetti, I. A., Aparicio, L. D. B., \& Cruz, C. (2016). Toxicidade aguda de inseticidas e espalhante usados no controle de vetores para o bioindicador caramujo (Pomacea canaliculata). Ciência e Cultura, 12(1), 45-51.

Liu, K., He, H., Shiji, X., Hu, L., Luo, K., Liu, X., Lui, M., Zhou, X., \& Bai, L. (2018). Mechanism of the effect of $\mathrm{pH}$ and biochar on the phytotoxicity of the weakacid herbicides imazethapyr and 2,4-D in soil to rice (Oryza sativa) and estimation by chemical methods. Ecotoxicology and Environmental Safety, 161, 602-609.

Miranda, G., Dalla Corte, C. L., Miranda, G. M., Leão, M. B., \& Moraes, P. M. (2017). Efeitos dos herbicidas picloram e 2,4-d sobre o estresse 
oxidativo em Drosophila melanogaster. In Anais do IX Salão Internacional de Ensino, Pesquisa e Extensão. Santana do Livramento: Universidade Federal do Pampa.

Nascimento, E. R., \& Yamashita, O. M. (2009). Desenvolvimento inicial de olerícolas cultivadas em solos contaminados com resíduos de 2,4-d + picloram. Semina: Ciências Agrárias, 30(1), 47-54.

Organization for Economic Co-operation and Development - OECD. (2009). Draft Guidance Document (4 p.). Paris: The Threshold Approach for Acute Fish Toxicity Testing.

Pereira, P. C., Brunetti, I. A., Castro, K. S., Chiarotti, L. F., Santos, B. E., Moraes, J. C., \& Cruz, C. (2019). Acute toxicity of herbicides and sensibility of aquatic plant Wolffia brasiliensis as a bioindicator organism. Planta Daninha, 37, e019201636.

Peres, L. R. S., Della Vechia, J. F., \& Cruz, C. (2017). Hormesis effect of herbicides subdoses on submerged macrophytes in microassay conditions. Planta Daninha, 35, e017165857.

Silva, A. F., Cruz, C. N., Neto, A., \& Pitelli, R. A. (2012). Ecotoxicidade de herbicidas para a macrófita aquática (Azolla caroliniana). Planta Daninha, 30(3), 541-546.

Tiburcio, R. A. S., Ferreira, F. A., Ferreira, L. R., Machado, M. S., \& Machado, A. F. L. (2012). Controle de plantas daninhas e seletividade do flumioxazin para eucalipto. Cerne, 18(4), 523-531.

United States Environmental Protection Agency USEPA. (2017). Techinical overview of ecological risk. Assessmet - Analysis Phase:Ecological Effects characterization. Recuperado em 2019, September 16, de https://www.epa.gov/ pesticide-science-and-assessing-pesticide-risks/ technical-overview-ecological-risk-assessment-0. 